\title{
Performance analysis of a Brayton Pumped Thermal Electricity Storage (PTES) with a liquid sensible heat storage
}

\author{
Guido Francesco Frate $^{1 *}$, Lorenzo Ferrari ${ }^{1}$, Luca Giachetti $^{1}$, Giacomo Petretto ${ }^{2}$ and Umberto Desideri ${ }^{1}$ \\ ${ }^{1}$ University of Pisa, Department of Energy, Systems, Territory and Construction Engineering (DESTEC), 56122 Largo Lucio \\ Lazzarino, Pisa, Italy \\ ${ }^{2}$ Enel S.p.A., Viale Regina Margherita 137, 00198 Roma, Italy
}

\begin{abstract}
A pervasive Renewable Energy Source (RES) exploitation poses a wide range of issues to electric grids, which the enhancement of the electric grid flexibility may mitigate. There are several approaches to improve grid flexibility, and a significant help will come from efficient, reliable, durable and cheap electric storage technologies. Inevitably, different storage technologies will be needed since different power and energy spectra characterise RES issues. In the category of high capacity-to-power ratio technologies, Pumped Thermal Electricity Storage (PTES) is becoming more and more popular. Such technology aims at replacing Pumped Hydro Energy Storage (PHES), and it is suited for daily cyclic operation (load shifting). In this paper, a Brayton PTES with liquid sensible heat storages is studied. Compared to the standard system equipped with packed beds, the liquid heat storage allows for a more straightforward state of charge estimation, control and, potentially, for a better usage of storage volume. Through a parametric analysis involving the main design specifications, the system performance is assessed under realistic assumptions. The resulting figures provide an exhaustive characterisation of the performance achievable by the system, which may be useful for a fair comparison between PTES and other competing storage technologies.
\end{abstract}

\section{Introduction}

The power production sector is a significant contributor to pollutants and green-house gasses emission. Therefore, substantial efforts must be promoted to decarbonise it. Such a task is done by changing the energy production mix and moving from a fossil fuelbased production to a widespread exploitation of Renewable Energy Sources (RESs). However, most of the RES growth potential is in solar and wind resources, which are fluctuating and challenging to dispatch.

Non-dispatchable RESs are non-flexible, whereas the electric grid must accommodate for the demand variations. Recent studies, e.g. [1], demonstrate that the required flexibility may come from various sources, among which energy storage.

Several energy storage technologies have been proposed to date. While electrochemical batteries dominate small capacity application niches, in the range of nominal power inputs and outputs of tens to hundreds of MW, and capacities around several hundreds of MWh (400 - $1000 \mathrm{MWh})$, several alternative technologies may compete. Such technologies, i.e. Compressed Air Energy Storage (CAES), Liquid Air Energy Storage (LAES), Pumped Thermal Electricity Storage (PTES), and others, try to inherit the role traditionally entrusted to Pumped Hydro Energy Storage (PHES). Therefore, all of these are cheap (capacity-wise), durable (20+ years), reliable and based on components (heat exchangers and turbomachines) which may effortlessly scale up in size.

The study focuses on the PTES, which is a leading contender among the listed technologies thanks to its geographical independence (as opposed to CAES) and the high achievable efficiency (as opposed to LAES).

Compared to other storage technologies, the peculiarity of PTES is to store the electric energy in the form of thermal energy [2]. The PTES configuration based on Brayton cycles will be investigated, which seem the most promising, efficiency-wise, compared to the Rankine-based one [3].

Brayton PTES is further divided into two categories, according to the use of solid or liquid materials for storing the thermal energy. Both the configurations have pros and cons and different achievable performance. Brayton PTES based on solid materials is by far the most studied, e.g. [4-6]. However, the configuration with liquid storage materials proposed in [7], may have some advantages, especially on the plant control side, and it is worthy of further investigation.

\footnotetext{
* Corresponding author: guidofrancesco.frate@ing.unipi.it
} 
In this study, the Brayton PTES configuration based on liquid materials will be analysed, and its performance will be calculated in terms of achievable efficiency and energy density. Furthermore, a performance sensitivity analysis is presented, by varying the most impactful parameters (i.e. turbomachine efficiency) to provide the reader with a comprehensive technology potentiality assessment. Therefore, the leading study contributions can be summarised as:

- A comprehensive performance assessment for a promising utility-scale storage technology is presented;

- A performance sensitivity analysis is provided to assess the impact of turbomachine performance.

\section{Liquid-based Brayton PTES}

\subsection{Operating principle}

In the paper, the Brayton PTES configuration with liquid storage materials described in [7] is taken as a reference. The plant layout is reported in Figure 1.

Even though PTES is often considered a power-toheat-to-power technology, the underlying operating principle is more precisely described in terms of thermal exergy. In the charge phase, a Brayton heat pump is operated, and the electric energy is used to move the heat from a cold to a hot reservoir (Figure 1). A temperature difference is established between the reservoirs. Therefore, what is stored is the potential of producing work by moving the heat backwards from the hot to the cold reservoir using a thermal engine. In other words, thermal exergy is stored. Such a point of view is especially useful since the cold reservoir is colder than the environment and heat is extracted from it. Nonetheless, the reservoir is charged. This is because at temperatures lower than the environment, exergy and heat flows have opposite direction, and, while the heat flows outside, the exergy flows inside.

To discharge a Brayton heat engine is used, as in Figure 1.

(a)

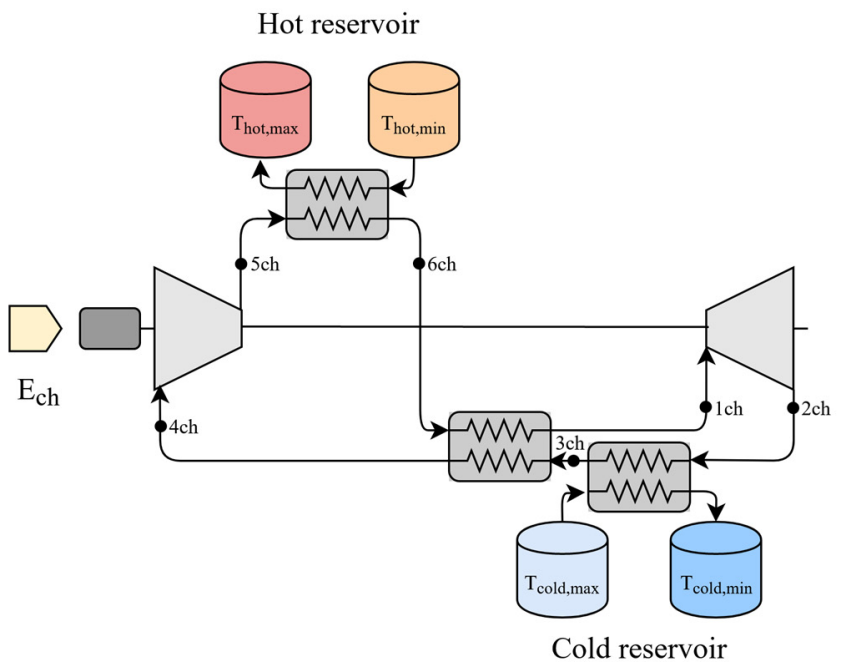

According to Figure 1, four machines and six heat exchangers must be used in the proposed Brayton PTES. Compared to the Brayton PTES with solid materials, the use of liquids requires introducing several heat exchangers. In "solid PTES", the hot and cold reservoirs are two regenerators, so the direct contact between the gas flowing in the machines and the storage material is used. Therefore, no heat exchangers are used, and higher performance can be achieved, thanks to the reduced operating temperature differences between operating fluid and storage materials. The use of regenerators yields better performances for a lower cost, compared to the use of liquid materials. However, the regenerator does not allow for an active control of the heat exchange. Thus, the solid material and gas outlet temperatures cannot be controlled for varying gas mass flowrates [8]. Such gas mass flow variations are dictated by the storage part-load operation, which may be frequent, depending on the utilisation context. For similar reasons, storage irregular operation may modify regenerator internal temperature distribution, such that the effect may reflect on a considerable number of subsequent cycles [8].

Differently, with liquid materials, mass flowrates on the reservoir side can be controlled to operate the charge and discharge always between the same temperatures [3]. In this way, each charge/discharge cycle operation is completely decoupled from the others, and the storage can freely operate.

Since the control easiness deriving from the use of liquids may counterbalance, in some operating contexts, the lower performance and higher costs, compared to the use of regenerators, liquid-based Brayton PTES is worth of further investigation.

As it is common for Brayton PTES, Argon is used as operating fluid. Given the operating temperature levels, molten salts (a mixture of $0.6 \mathrm{NaNO}_{3}$ and $0.4 \mathrm{KNO}_{3}$ ) are used in the hot reservoir and liquid Hexane in the cold reservoir.

Thermophysical properties of fluids are calculated by means of CoolProp [9].

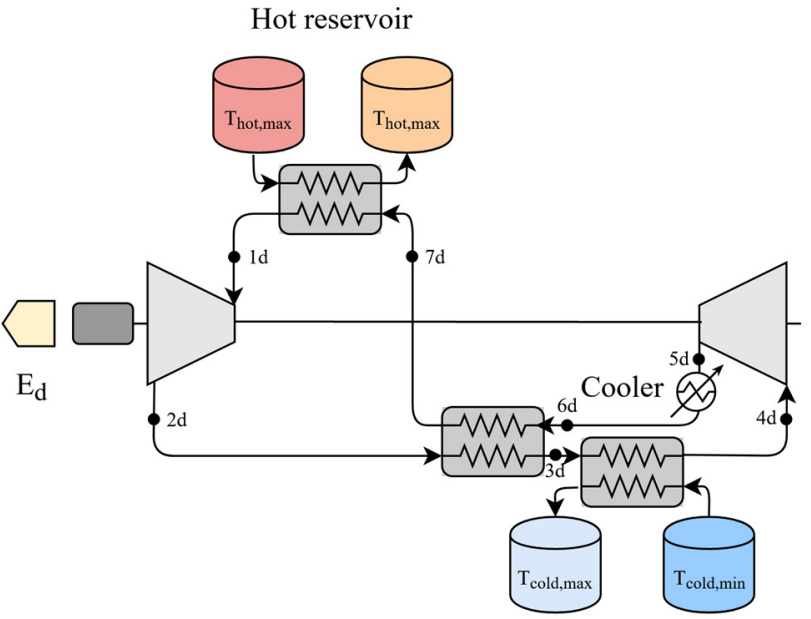

Cold reservoir

Figure 1. Brayton PTES system with liquid-based thermal exergy storage. (a): charge system; (b): discharge system. 


\subsection{Optimised plant design}

An optimisation problem is solved to identify the pressure and temperature configurations that maximise the plant roundtrip efficiency $\varepsilon_{r}$, defined as the ratio between discharged and charged amount of electric energy. The charge and discharge processes are in series, and the electric energy is alternatively converted into thermal exergy and vice versa. Therefore, $\varepsilon_{r t}$ may be written with respect to charge and discharge exergy efficiencies, i.e. $\phi_{c h}$ and $\phi_{d}$, respectively, as in Eq. 1:

$$
\varepsilon_{r t}=\frac{E_{d}}{E_{c h}}=\phi_{c h} \cdot \phi_{d}
$$

$\phi_{c h}$ and $\phi_{d i s}$ are defined as in Eq 2 and 3, respectively:

$$
\begin{aligned}
& \phi_{c h}=\frac{E x_{\text {hot }}+E x_{\text {cold }}}{E_{c h}} \\
& \phi_{d}=\frac{E_{d}}{E x_{\text {hot }}+E x_{\text {cold }}}
\end{aligned}
$$

Where, $E x_{\text {hot }}$ and $E x_{\text {cold }}$ are the stored thermal exergy in the hot and cold reservoir, respectively.

The optimisation problem may be formally written as in Eq. 4:

$$
\underset{\boldsymbol{x} \in \mathrm{F} \subseteq \mathbb{R}^{m}}{\operatorname{Maximise}} \varepsilon_{r t}(\boldsymbol{x})
$$

Where $\boldsymbol{x}$ is the optimisation variable vector, $F$ is the feasible region, the $\mathbb{R}^{m}$ subset defined by the problem constraints, and $m$ is the number of optimisation variables. By referring to Figure 2, the $\boldsymbol{x}$ elements are the charge and discharge cycle temperatures $\left(T_{x c h}\right.$ and $\left.T_{x d}\right)$ and pressure ratios $\left(\beta_{c h}\right.$ and $\left.\beta_{d}\right)$ (Eq. 5):

$$
\boldsymbol{x}=\left\{T_{1 c h} \ldots T_{6 c h}, T_{1 d} \ldots T_{7 d}, \beta_{c h}, \beta_{d}\right\}
$$

The feasible region $F$ is expressed through the equation and inequality system in Eq. 6 to 15 and 16 to 24 , for the charge and discharge, respectively:

$$
\begin{aligned}
& T_{1 c h}=T_{2 c h} \cdot\left(\beta_{c h}\right)^{\frac{k-1}{k \cdot \eta p, c m p}} \\
& T_{4 c h}=T_{5 c h} \cdot\left(\beta_{c h}\right)^{\left(-\eta_{p, \text { exp }} \cdot \frac{k-1}{k}\right)} \\
& h_{2 c h}-h_{3 c h}=h_{6 c h}-h_{5 c h} \\
& T_{1 c h} \geq T_{h o t, \text { max }}+p p_{\text {hot }} \\
& T_{6 c h} \geq T_{\text {hot,min }}+p p_{\text {hot }} \\
& T_{4 c h} \leq T_{\text {cold,min }}-p p_{\text {cold }} \\
& T_{3 c h} \leq T_{\text {cold,max }}-p p_{\text {cold }} \\
& T_{6 c h} \geq T_{2 c h}+p p_{\text {reg }} \\
& T_{5 c h} \geq T_{3 c h}+p p_{\text {reg }} \\
& T_{1 c h} \leq T_{\text {max }, \text { cmp }}
\end{aligned}
$$

Where Eq. 6 and 7 are the constitutive equation of the compression and expansion process, with $\eta_{p, c m p / e x p}$ being the compressor and expander polytropic efficiency; Eq. 8 is the heat balance on the charge cycle regenerator; Eq. 9 to 12 enforce the observance of pinch points $\left(p p_{\text {hot } c o l d r e g}\right)$ in the heat exchangers of the hot and cold reservoirs and the regenerator. As reported in Figure 1, $T_{\text {hot/cold, max }}$ and $T_{\text {hot/cold,min }}$ represent the hot and cold reservoir operating temperatures. Finally, Eq 15 limit the compressor discharge temperature to a maximum value to be set based on the considered technology limit. Modern aero-derivative turbine compressors may achieve a discharge temperature around $600{ }^{\circ} \mathrm{C}$, which should be considered the current technology limitation for such components.

As for the discharge:

$$
\begin{aligned}
& T_{5 d}=T_{4 d} \cdot\left(\beta_{d}\right)^{\frac{k-1}{k \cdot \eta, c m p}} \\
& T_{2 d}=T_{1 d} \cdot\left(\beta_{d}\right)^{\left(-\eta_{p, e x p} \cdot \frac{k-1}{k}\right)} \\
& h_{2 d}-h_{3 d}=h_{7 d}-h_{6 d} \\
& T_{1 d} \leq T_{\text {hot,max }}+p p_{\text {hot }} \\
& T_{7 d} \leq T_{\text {hot,min }}+p p_{\text {hot }} \\
& T_{4 d} \geq T_{\text {cold,min }}-p p_{\text {cold }} \\
& T_{3 d} \geq T_{\text {cold,max }}-p p_{\text {cold }} \\
& T_{7 d} \leq T_{2 d}-p p_{\text {reg }} \\
& T_{6 d} \geq T_{3 d}-p p_{\text {reg }}
\end{aligned}
$$

The previous equations have a similar meaning to that discussed for the charge phase.

By solving the problem in Eq. (4), the configuration that maximises the roundtrip efficiency given a set of boundary conditions can be found. Such boundary conditions (i.e. the constraints) are affected by several numeric parameters, like the polytropic machine efficiency and heat exchangers pinch points, which are to be specified in advance. The numeric values assumed for such parameters are reported in Table 1.

The other important KPI, the exergy density $\rho_{e x}$, defined as in Eq. 25, is not directly involved in the optimisation process, but it is monitored for each resulting configuration. Such parameter measures the total reservoir size for each stored $k W h$, and it is a relevant parameter from the economic point of view (the lower the volume, the lower the cost per $k W h$ ). $\rho_{e x}$ can be written as (Eq. 25):

$$
\rho_{e x}=\frac{E_{c h} \cdot \varepsilon_{r t}}{V_{t o t}}=\frac{E_{c h} \cdot \varepsilon_{r t}}{V_{h o t}+V_{c o l d}}
$$

Where $V_{\text {tot }}=V_{\text {hot }}+V_{\text {cold }}$ is the total volume occupied by the hot and cold reservoirs.

It is interesting to notice that, since only electric inputs and outputs are involved, exergy density $\rho_{e x}$ is entirely equivalent to energy density.

Based on the ranges reported in Table 1, a sensitivity analysis is performed on the performance achievable by the Brayton PTES system investigated in the paper.

For all the configurations, the hot and cold reservoir operating temperatures are imposed as a boundary condition and are not optimised. The assumed values can be found in Table 2 .

\section{Results and discussion}

The reference Brayton PTES configuration resulting from the problem in Eq. (4) is reported in Figure 2. The compressor discharge temperature is limited to $T_{\text {max }}$, cmp $=500{ }^{\circ} \mathrm{C}$ and the polytropic machine efficiencies $\left(\eta_{p, c m p / \text { exp }}\right)$ are assumed to be equal to 0.87 and 0.92 , respectively, for the reported case. 


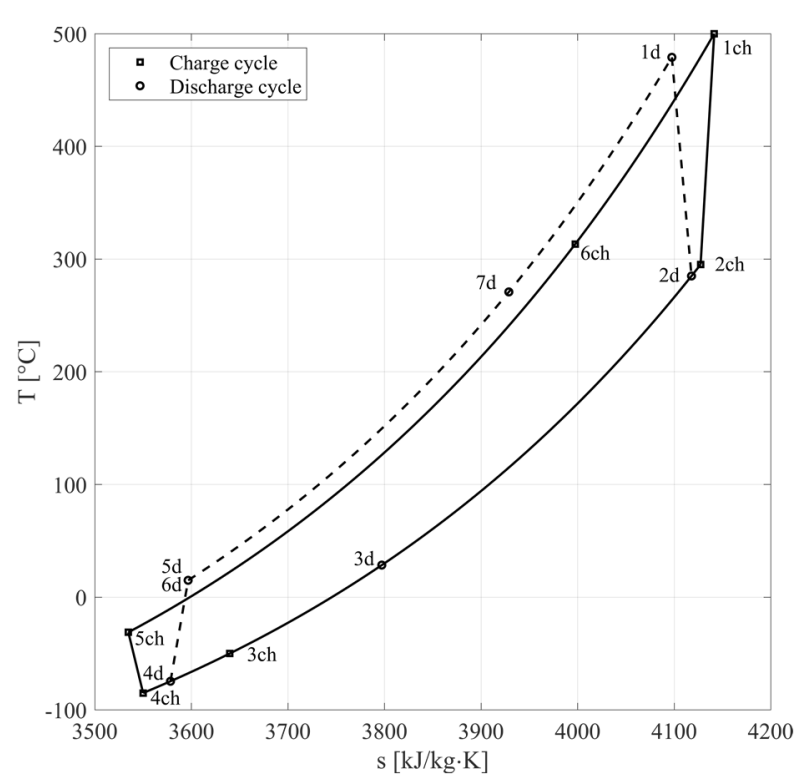

Figure 2. Brayton PTES system thermodynamic cycles during charge (ch) and discharge $(d)$.

Table 1. Parameter numeric values.

\begin{tabular}{|c|c|c|c|}
\hline Parameter & $\begin{array}{c}\eta_{p, \text { cmplexp }} \\
{[-]}\end{array}$ & $\begin{array}{c}\text { pphot/cold/reg } \\
{[\mathrm{K}]}\end{array}$ & $\begin{array}{c}T_{\max , \text { cmp }} \\
{\left[{ }^{\circ} \mathrm{C}\right]}\end{array}$ \\
\hline Value & $0.85-0.95$ & 5 & $400-600$ \\
\hline
\end{tabular}

Table 2. Hot and cold reservoir operating temperatures.

\begin{tabular}{|c|c|c|c|c|}
\hline Parameter & $\begin{array}{c}T_{\text {hot,max }} \\
{\left[{ }^{\circ} \mathrm{C}\right]}\end{array}$ & $\begin{array}{c}T_{\text {hot,min }} \\
{\left[{ }^{\circ} \mathrm{C}\right]}\end{array}$ & $\begin{array}{c}T_{\text {cold,max }} \\
{\left[{ }^{\circ} \mathrm{C}\right]}\end{array}$ & $\begin{array}{c}T_{\text {cold,min }} \\
{\left[{ }^{\circ} \mathrm{C}\right]}\end{array}$ \\
\hline Value & $390-590$ & 300 & 15 & -80 \\
\hline
\end{tabular}

The cooler in the discharge cycle in Figure 1 (b) is used for rejecting into the environment the heat generated from irreversibilities, and it is always included in PTES layouts [3].

However, for the same hot and cold reservoir operating temperatures, the thermodynamic cycle arrangement is not unique, and it depends on the design methodology. In this case, an optimised design methodology is used, and the cooler usage is minimised, thus maximising the storage performance.

In Figure 3 (a), the PTES system roundtrip efficiency can be found. In the reference configuration, $\varepsilon_{r t}=0.50$, which could be considered a satisfying value depending on the utilisation context and the investigated technology-specific cost. However, these additional aspects are not addressed in the paper and are postponed to future analyses.

As far as the energy density is concerned, in Figure 3 (b), it can be found that $\rho_{e x}=18.2 \mathrm{kWh} / \mathrm{m}^{3}$.

Both the achieved roundtrip efficiency and energy density are significantly lower than those which characterises the Brayton PTES based on solid materials. Even though the liquid-based PTES could have higher performance than the solid-based one, in theory, the use of finite temperature differences in the heat exchangers introduces significant exergy losses [3]. The impact of such losses is relevant, and the roundtrip efficiency of the systems with solid materials can be around 0.6 , e.g. $[3,4]$, and the energy density can be higher than the one resulting in Figure 3 (a), [5].

For what concerns the sensitivity analysis, in Figure 3 (a) and (b) the sensitivity of $\rho_{e x}$ and $\varepsilon_{r t}$ to $\eta_{p, c m p}$ and $\eta_{p, e x p}$ can be found. As it resulted, $\varepsilon_{r t}$ increased almost linearly with both $\eta_{p, c m p}$ and $\eta_{p, \text { exp }}$. It must be considered that the reference configuration is calculated with $\eta_{p, c m p}$ $=0.87$ and $\eta_{p, \text { exp }}=0.92$, which should be considered representative of modern turbomachines performance. Realistically, the PTES technology cannot achieve much higher performance, as only marginal improvements on the machine performance should be expected soon in the future. On the other hand, worse machine performance may easily result due to the absence of dedicated designs (the machines operate with argon and in non-standard conditions) and off-design operation. In these cases, $\varepsilon_{r t}$ comprised between 0.45 and 0.5 may easily result. Such roundtrip efficiency values could make the technology uneconomical. Therefore, the availability of dedicated high-quality machine is a mandatory prerequisite to the widespread of Brayton PTES.

The $\rho_{e x}$ relationship with $\eta_{p, c m p}$ and $\eta_{p, e x p}$ is similar to that of $\varepsilon_{r t}$, but the relative increments are much lower. $\rho_{e x}$ is mainly linked to discharge efficiency $\phi_{d}$, and it increases when $\phi_{d}$ improves. Such a trend arises because the exergy stored in the volume $\left(V_{t o t}\right)$ is more efficiently converted if the system operates with better $\phi_{d}$.

On the other hand, if $\phi_{c h}$ improves, $\varepsilon_{r t}$ increases, which is still beneficial for $\rho_{e x}$. However, by reducing the charge cycle irreversibilities, not only $\phi_{c h}$ improves, but also the ratio between the heat moved from the cold to hot reservoir and the absorbed electric energy, i.e. the $C O P$ of the charge cycle, which operates as a heat pump. Since more thermal energy is stored for the same electric input, larger volumes $V_{t o t}$ are required, and the energy density tends to decrease. The second effect (the increment of $V_{\text {tot }}$ ) counterbalances the first (the increment of $\varepsilon_{r t}$ ) and $\rho_{e x}$ increases with machine polytropic efficiencies less than $\varepsilon_{r t}$.

For the same reason, the $\rho_{e x}$ variation range with $\eta_{p, e x p}$ and $\eta_{p, c m p}$, is more limited than that of $\varepsilon_{r}$, and the achieved values are always in the order of $18 \mathrm{kWh} / \mathrm{m}^{3}$, which is a good value, compared to other storage technologies like PHES and CAES, which are characterised by densities in the order of $1 \mathrm{kWh} / \mathrm{m}^{3}[10]$. Furthermore, the achieved $\rho_{e x}$ values are significantly higher than the values usually achieved by Rankine PTES systems [11].

Analogous considerations may be drawn for the impact of $T_{\text {max }, \text { cmp }}$ on $\varepsilon_{r t}$ and $\rho_{e x}$. By reducing the maximum cycle temperature $\varepsilon_{r t}$ decreases. As explained in [3], this is because the irreversibility relative impact increases as the average temperature difference between the thermal reservoirs is reduced. From the energy point of view, as $T_{\max , c m p}$ is reduced, the charge cycle $C O P$ increases, so more thermal energy is charged. However, this does not translate into an increment of $\phi_{c h}$, which increases with $T_{\max , \text { cmp }}$, instead.

The same trend is followed by $\phi_{d}$, which increases with $T_{\max , c m p}$ according to the traditional behaviour of heat engine cycles. As a result, since both $\phi_{c h}$ and $\phi_{d}$ 
increases with $T_{\max , c m p}$, to maximise $\varepsilon_{r t}$, the maximum possible operating temperatures should be adopted.

As Figure 3 (c) shows, by increasing $T_{\max , \text { cmp }}$ from $400{ }^{\circ} \mathrm{C}$ to $500{ }^{\circ} \mathrm{C}$ yields significant efficiency improvements, particularly if the system operates with very high machine polytropic efficiencies. Further increments, e.g. from $500{ }^{\circ} \mathrm{C}$ to $600{ }^{\circ} \mathrm{C}$, may bring additional advantages, but the relative increment might be marginal especially if the system operates with machines with low polytropic efficiencies. In analogy with what found for Brayton PTES with solid materials in [4], Figure 3 (c) demonstrates how higher operating temperatures could partly balance the use of lowefficiency machines.

Finally, in Figure 3 (d), the impact of $T_{\max , \text { cmp }}$ on the exergy density can be observed. Similarly to what happens for $\varepsilon_{r t}, \rho_{e n}$ increases for higher $T_{\max , c m p}$. The explanation for this is again in the behaviour of charge and discharge exergy efficiencies and charge cycle COP.

As $T_{\max , \text { cmp }}$ decreases, $\phi_{c h}, \phi_{d}$ and, thus, $\varepsilon_{r t}$ decreases, which tends to lower $\rho_{e x}$, according to Eq. (25). Furthermore, $V_{\text {tot }}$ is sized according to the stored amount of thermal energy, which depends on the charge cycle $C O P$. Such a parameter increases as $T_{\max , c m p}$ decreases because the average temperature difference between the thermal reservoirs is reduced, and less work is needed to move the heat between the two. Therefore, for the same amount of $E_{c h}$, a larger volume $V_{\text {tot }}$ is needed to accommodate the stored thermal energy. Even though the amount of stored energy is increased, its average temperature is diminished due to lower $T_{\max , c m p}$. Therefore, less exergy is stored, as the $\phi_{c h}$ reduction testimonies. The two effects, the reduction of $V_{t o t}$ and the increment of $\varepsilon_{r t}$, sum up and the increment of $\rho_{e x}$ with $T_{\max , \mathrm{cmp}}$ is even stronger than that resulting for $\varepsilon_{r t}$.

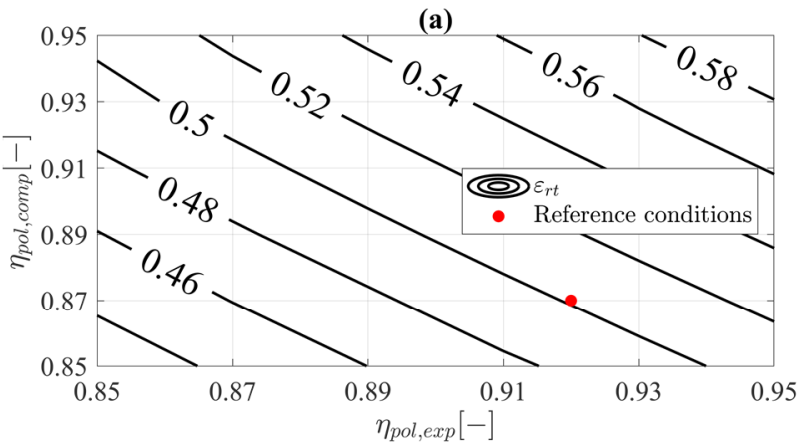

(c)

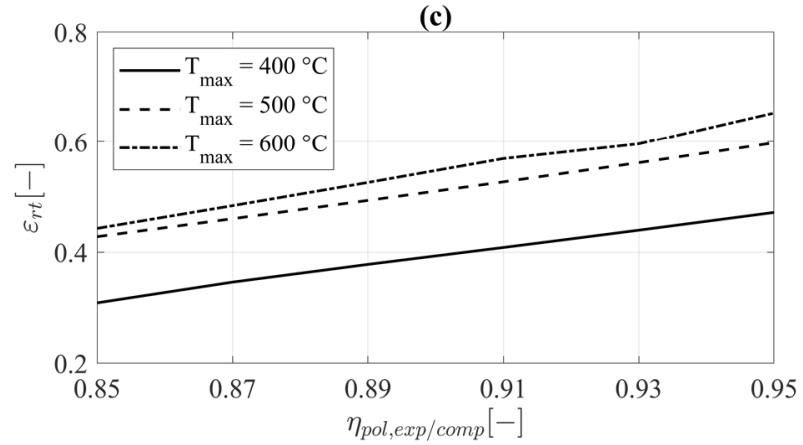

In a similar way to what observed for $\varepsilon_{r t}$, relevant $\rho_{e x}$ increments can be achieved by incresing $T_{\max , \mathrm{cmp}}$ from $400{ }^{\circ} \mathrm{C}$ to $500{ }^{\circ} \mathrm{C}$, with a slightly additional advantages if machines with high polytropic efficiencies are used. Instead, by increasing $T_{\max , c m p}$ from $500{ }^{\circ} \mathrm{C}$ to $600{ }^{\circ} \mathrm{C}$, smaller improvements may be achieved. This implies that the $T_{\max , \mathrm{cmp}}$ maximisation may be used as a strategy to improve both $\rho_{e x}$ and $\varepsilon_{r t}$ (to reduce the cost, or increasing the performance in general), if significant improvements on the machine side cannot be achieved.

Even in this case, a relevant technology improvement must be pursued and achieved to overcome the current technological limitations on $T_{\text {max }, \text { cmp }}$.

\section{Conclusion}

In the paper, a Brayton PTES system with liquidbased thermal sensible heat storage is studied. Firstly, an optimised design procedure is presented. By formulating the thermodynamic system design as an optimisation problem, the maximum roundtrip efficiency configuration is selected. The same optimisation problem is solved several times based on different sets of optimisation constraints. In this way, a sensitivity analysis was performed to identify the impact of polytropic machine efficiency and maximum cycle operating temperatures. As it resulted, in the reference configuration, the investigated storage technology may achieve a roundtrip efficiency $\varepsilon_{r t}$ in the order of around 0.5 and an energy density $\rho_{e n}$ around $18.2 \mathrm{kWh} / \mathrm{m}^{3} . \varepsilon_{r t}$ was found to increase with both compressor and expander polytropic efficiency.

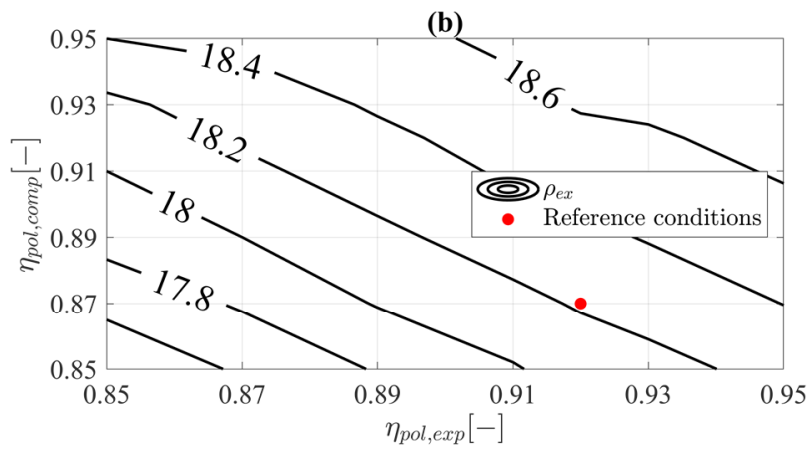

(d)

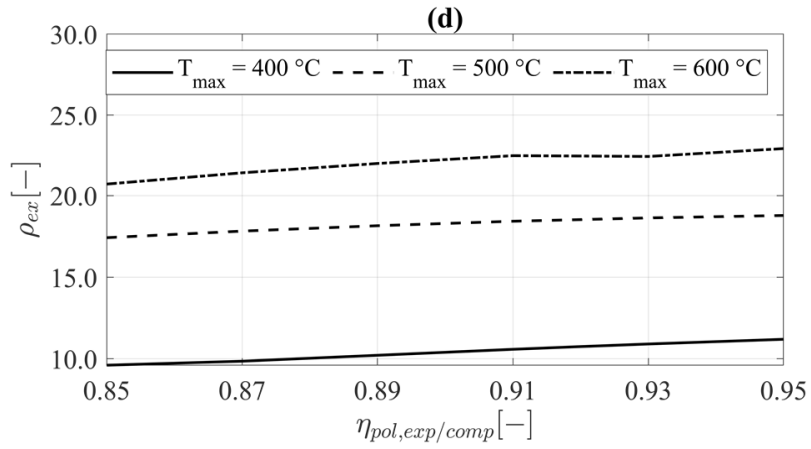

Figure 3. Sensitivity analysis results. (a): $\varepsilon_{r t}$ with respect to $\eta_{p, c m p}$ and $\eta_{p, \exp }$. (b): $\rho_{e x}$ with respect to $\eta_{p, c m p}$ and $\eta_{p, \exp }$. (c): $\varepsilon_{r t}$ with respect to $\eta_{p, c m p}=\eta_{p, \text { exp }}$ and $T_{\max , \text { cmp. }}$ (d): $\rho_{\text {ex }}$ with respect to $\eta_{p, c m p}=\eta_{p, \text { exp }}$ and $T_{\max , c m p}$. 
While $\varepsilon_{r t}$ around 0.60 may be unfeasible, since the required polytropic efficiencies would be too high, $\varepsilon_{r t}$ might easily fall under 0.50 if non-specialised machine designs are used and the resulting polytropic efficiencies are between $0.85-0.89$.

Differently, $\rho_{e n}$ have a narrower variation range, and the resulting values are all in the order of $18 \mathrm{kWh} / \mathrm{m}^{3}$ and comprised between 17.6 to $18.6 \mathrm{kWh} / \mathrm{m}^{3}$.

Finally, the impact of maximum operating temperatures was assessed. High operating temperatures improve $\varepsilon_{r t}$ and can partly counterbalance the effect of low polytropic efficiencies. However, passing from 500 ${ }^{\circ} \mathrm{C}$ to $600{ }^{\circ} \mathrm{C}$, i.e. the current technological limit for compressors, may only yield marginal improvements. Therefore, $\varepsilon_{r t}$ values not much higher than 0.5 should be expected soon in the future.

By increasing the maximum operating temperatures, $\rho_{e x}$ is improved as well, especially if high polytropic efficiencies are adopted. In this case, given the lesser impact of machine efficiencies on $\rho_{e x}$, resorting on high operating temperatures is the most impactful mechanism to reduce the volume occupied by the system.

\section{References}

[1] Zappa W, Junginger M, van den Broek M. Is a $100 \%$ renewable European power system feasible by 2050? Appl Energy 2019;233234:1027-50.

doi:10.1016/j.apenergy.2018.08.109.

[2] Benato A, Stoppato A. Pumped Thermal Electricity Storage: A technology overview. Therm Sci Eng Prog 2018;6:301-15. doi:10.1016/j.tsep.2018.01.017.

[3] Steinmann W-D, Jockenhöfer H, Bauer D. Thermodynamic Analysis of HighTemperature Carnot Battery Concepts. Energy Technol 2020;8:1900895. doi:10.1002/ente.201900895.

[4] Desrues T, Ruer J, Marty P, Fourmigué JF. A thermal energy storage process for large scale electric applications. Appl Therm Eng 2010;30:425-32. doi:10.1016/j.applthermaleng.2009.10.002.

[5] Ni F, Caram HS. Analysis of pumped heat electricity storage process using exponential matrix solutions. Appl Therm Eng 2015;84:3444. doi:10.1016/j.applthermaleng.2015.02.046.

[6] McTigue JD, White AJ, Markides CN. Parametric studies and optimisation of pumped thermal electricity storage. Appl Energy 2015;137:800-11.

doi:10.1016/j.apenergy.2014.08.039.

[7] Laughlin RB. Pumped thermal grid storage with heat exchange. J Renew Sustain Energy 2017;9:044103. doi:10.1063/1.4994054.

[8] McTigue JD, Markides CN, White AJ. Performance response of packed-bed thermal storage to cycle duration perturbations. J Energy Storage 2018;19:379-92. doi:10.1016/j.est.2018.08.016.

[9] Bell IH, Wronski J, Quoilin S, Lemort V. Pure
andPseudo-pure Fluid Thermophysical Property Evaluation and the OpenSourceThermophysical Property Library CoolProp. Ind Eng Chem Res 2014;53:2498508. doi:dx.doi.org/10.1021/ie4033999.

[10] Frate GF, Ferrari L, Desideri U. Critical review and economic feasibility analysis of electric energy storage technologies suited for grid scale applications. E3S Web Conf 2019;137:01037. doi:10.1051/e3sconf/201913701037.

[11] Frate GF, Ferrari L, Desideri U. Multi-criteria investigation of a pumped thermal electricity storage (PTES) system with thermal integration and sensible heat storage. Energy Convers Manag 2020;208:112530. doi:10.1016/j.enconman.2020.112530. 